\title{
Fondation de la F2S, Fédération Françuise des Sociétés Scientifiques
}

La Société Française de Physique vient de faire un nouveau pas : elle s'allie à deux autres sociétés savantes pour fonder la F2S, ou "Fédération Française des Sociétés Scientifiques". Ses deux partenaires sont la SFO, Société Française d'Optique, et la SEE, Société de l'Électricité, de I'Électronique et des Technologies de l'Information et de la Communication. D'autres sociétés sont susceptibles de rejoindre la F2S prochainement ; toutefois les membres fondateurs préfèrent une association de taille réduite qui fasse ses preuves avant de s'élargir. L'idée est d'associer le monde de la recherche universitaire, représenté par la SFP et une partie de la SFO, à celui des ingénieurs adhérents de la SEE ou de la SFO. La démarche de constitution de la F2S s'inspire de celle des chimistes qui ont créé la FFC, Fédération Française pour les sciences de la Chimie, en 2005 sous l'impulsion d'Armand Lattes, alors président de la Société Française de Chimie (actuelle SCF). Le siège de la F2S est d'ailleurs situé à la Maison de la Chimie (rue Saint-Dominique à Paris), ce qui devrait favoriser des entreprises communes avec les chimistes pour la maîtrise des grands défis de société, tels l'environnement, I'énergie ou la pollution.

La F2S, surtout après son élargissement programmé pour les années futures, devrait ainsi mieux représenter l'ensemble des scientifiques travaillant dans le champ de la physique et de ses applications. Le total actuel des adhérents des trois sociétés fondatrices est de I'ordre de 8000. À terme, la F2S devrait atteindre un poids approchant celui des sociétés de physique anglaise ou allemande. Les interlocuteurs visés sont d'abord les décideurs politiques à l'échelle de la France, en cette période de réflexion et de réformes constantes dans les universités, la recherche et I'enseignement secondaire. La F2S a aussi I'ambition d'être porte-parole des sciences physiques françaises auprès des instances européennes et au plan international. Elle souhaite enfin jouer un rôle pour la promotion de la culture scientifique, en particulier en fournissant des informations aux médias sur les sujets d'intérêt sociétal.
D'une façon générale, la F2S souhaite développer des actions en faveur des sciences et de leurs applications, et tout particulièrement des métiers qui y sont associés. Il y a un grand défaut d'information auprès des jeunes, relatif à la variété et à l'intérêt des carrières fondées sur une formation initiale de physique. Les besoins des entreprises sont également mal connus et souvent mal perçus. La F2S sera partenaire de la réflexion sur les contenus de l'enseignement secondaire et sur la formation initiale et continue des enseignants. Elle ambitionne aussi d'être un interlocuteur sur toutes sortes de questions d'expertise ou d'éthique scientifiques. Elle organisera des débats, la remise de plusieurs prix, ainsi que des colloques nationaux ou internationaux, parfois à la demande des industriels. Elle sera aussi un moteur pour la promotion des sciences vers le grand public : le premier exemple sera fourni par les manifestations de l'anniversaire des 50 ans du laser en 2010.

Les membres du bureau actuel de la F2S sont Alain Bravo (SEE), Claude Fabre (SFO) et moi-même (SFP). Un vice-président issu du milieu industriel sera adjoint prochainement. Un site web sera tenu à jour et des moyens seront trouvés auprès de mécènes et par l'organisation de congrès.

À titre personnel, je suis très intéressée par cette nouvelle activité qui va prolonger mon travail pour la SFP, à l'issue d'un mandat exceptionnellement long de trois ans comme présidente. Après les circonstances qui ont secoué notre société au printemps 2009, j'ai bon espoir de laisser une SFP vivante, lieu de riches débats et œuvrant pour la vitalité de la discipline. Je suis convaincue que la création de la F2S ne pourra que renforcer l'attractivité de la SFP, en élargissant son champ d'action et son audience.

Michèle Leduc

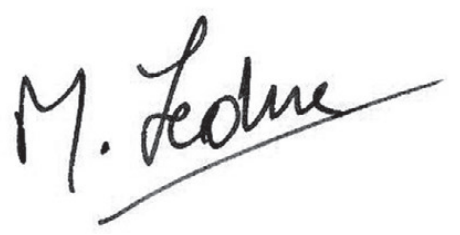

